\title{
First and Second Quantised Neutron Diffusion Equations
}

\author{
E. B. Davies \\ St. John's College, Oxford OX1 3JP, England
}

\begin{abstract}
We show that a quantised linear Boltzmann equation can be obtained as an exact contracted form of a second quantised neutron diffusion equation in the weak coupling limit.
\end{abstract}

\section{§ 1. Introduction}

Let $\mathscr{F}$ be the fermion Fock space built from a single particle space $\mathscr{H}=L^{2}\left(\mathbb{R}^{3}\right)$ and consider the evolution equation

$$
\varrho^{\prime}(t)=-i[H, \varrho]-\lambda(R \varrho+\varrho R) / 2+\lambda J(\varrho),
$$

where $\varrho(t)$ lies in the space $V=\mathscr{T}_{s}(\mathscr{F})$ of self-adjoint trace-class operators on $\mathscr{F}$. Here $H$ is the free Hamiltonian on $\mathscr{F}$ which equals $-\Delta$ on $\mathscr{H}$. The unbounded positive linear map $J: V \rightarrow V$ is defined by

$$
J(\varrho)=\int_{\mathbb{R}^{3}} B_{x} \varrho B_{x}^{*} d x
$$

where

$$
B_{x}=a^{*}\left(f_{x}^{3}\right) a^{*}\left(f_{x}^{2}\right) a\left(f_{x}^{1}\right) .
$$

In Equation (1.3), $a$ and $a^{*}$ are the fermion field operators smeared by the test functions $f_{x}^{i}$ in Schwartz space $\mathscr{S}$. Moreover $f_{x}^{i}$ is defined as the translate by a distance $x \in \mathbb{R}^{3}$ of $f^{i}$ and it is supposed that $f^{2}$ and $f^{3}$ have disjoint supports in momentum space. Finally $\lambda>0$ and

$$
R=\int_{\mathbb{R}^{3}} B_{x}^{*} B_{x} d x .
$$

Without further loss of generality we suppose that $f^{2}$ and $f^{3}$ have unit norm in $\mathscr{H}$.

The above phenomenological evolution equation describes a variable number of neutrons moving in a translation invariant external reservoir of unstable atoms, which they can induce to decay emitting further neutrons. It is shown in [2] that Equation (1.1) has the solution

$$
\varrho(t)=T_{t}\{\varrho(0)\}
$$


where $T_{t}$ is a strongly continuous, positive, trace-preserving semigroup on $V$. We shall generally not exhibit the dependence of semigroups on the parameter $\lambda$.

The same physical problem has a technically much simpler formulation which does not involve second quantisation. One starts from the evolution equation

$$
\sigma^{\prime}(t)=-i[H, \sigma]-\lambda(A \sigma+\sigma A) / 2+\lambda K(\sigma)
$$

where $\sigma(t)$ lies in $W=\mathscr{T}_{s}(\mathscr{H})$. The bounded operator $K: W \rightarrow W$ is given by

$$
K=K_{2}+K_{3}
$$

where

$$
K_{i}(\sigma)=\int_{\mathbb{R}^{3}}\left|f_{x}^{i}\right\rangle\left\langle f_{x}^{i}\right|\left\langle\sigma f_{x}^{1}, f_{x}^{1}\right\rangle d x .
$$

The operator $A$ on $\mathscr{H}$ is defined by

$$
A=\int_{\mathbb{R}^{3}}\left|f_{x}^{1}\right\rangle\left\langle f_{x}^{1}\right| d x
$$

and we are using the symbol $H$ to denote the self-adjoint operator $-\Delta$ on $\mathscr{H}$ as well as the free Hamiltonian on $\mathscr{F}$. We shall show that Equation (1.6) has the solution

$$
\sigma(t)=D_{t}\{\sigma(0)\}
$$

where $D_{t}$ is a strongly continuous, positive, one-parameter semigroup on $W$, which is not, however, trace-preserving. Indeed according to $[2], \operatorname{tr}[\sigma(t)]$ is an increasing function of $t$ which represents the number of particles at time $t$.

In order to relate the above two semigroups we introduce the Banach spaces

$$
V_{n}=\left\{\varrho \in V:\|\varrho\|_{n} \equiv \operatorname{tr}\left[(1+N)^{n}|\varrho|\right]<\infty\right\}
$$

where $N$ is the number operator on $\mathscr{F}$. The equation

$$
\langle(P \varrho) \phi, \psi\rangle=\operatorname{tr}\left[a^{*}(\phi) a(\psi) \varrho\right]
$$

where $\phi, \psi \in \mathscr{H}$, defines a positive linear map $P: V_{1} \rightarrow W$ which is a contraction, and a projection if we regard $W$ as a subspace of $V_{1}$ in the obvious manner.

The two dynamical semigroups are related by comparing $P T_{t} \varrho$ with $D_{t} \varrho$ when $\varrho \in W$. We show that they become asymptotically equal as $\lambda \rightarrow 0$ in the sense that for all $\tau_{0} \geqq 0$

$$
\lim _{\lambda \rightarrow 0}\left\{\sup _{0 \leqq \lambda t \leqq \tau_{0}}\left\|P T_{t} \varrho-D_{t} \varrho\right\|\right\}=0 .
$$

As $\lambda \rightarrow 0$ the time $t$ is allowed to become longer so that the accumulated effects of the stochastic terms $J$ and $K$ are non-zero.

Results of the type of Equation (1.13) may be found in $[1,3,7,8]$, but our problem requires some new ideas. In Section 2 we explain the need for these and state the main result of the paper. The associated diagrammatic analysis is given in Section 3.

We finally remark that although technically very different, our model has a very similar statistical mechanical significance to one studied by Kac and McKean [4-6]. 


\section{§ 2. The Main Results}

Our first technical problem can be explained most simply by examining the analogous pure birth process of probability theory. In the space $l^{1}\left(\mathbb{Z}^{+}\right)$of absolutely summable sequences on the non-negative integers $\mathbb{Z}^{+}$the FokkerPlanck equation

$$
f_{n}^{\prime}(t)=-\lambda(n+1) f_{n}(t)+\lambda n f_{n-1}(t)
$$

with $f_{0}(0)=1$ and $f_{n}(0)=0$ for $n>0$, has the solution

$$
f_{n}(t)=e^{-\lambda t}\left(1-e^{-\lambda t}\right)^{n} .
$$

One sees that $f(t) \in l^{1}\left(\mathbb{Z}^{+}\right)$for all $t \geqq 0$. If however one expands $f$ in powers of $\lambda t$ one finds that the series, which has coefficients in $l^{1}\left(\mathbb{Z}^{+}\right)$, has a finite radius of convergence. This suggests that one can only hope to derive Equation (1.13) by direct expansion of both terms in powers of $\lambda$ if $\tau_{0}$ is sufficiently small (a similar difficulty arose in [7]). As in quantum field theory the difficulty is circumvented by resummation and use of positivity.

We use the notation and results of [2] to obtain some properties of the oneparameter semigroup $T_{t}$ on $V$.

Lemma 2.1. The semigroup $T_{t}$ leaves each of the subspaces $V_{n}$ invariant, and there are constants $c_{n}$ such that

$$
\left\|T_{t} \varrho\right\|_{n} \leqq e^{c_{n} \lambda t}\|\varrho\|_{n}
$$

for all $\varrho \in V_{n}$ and all $\lambda, t, n \geqq 0$.

Proof. It is sufficient to construct a semigroup $T_{{ }_{t}}^{\sim}$ on $V$ such that

$$
(N+1)^{-n / 2} T^{\sim}{ }_{t}(\varrho)(N+1)^{-n / 2}=T_{t}\left\{(N+1)^{-n / 2} \varrho(N+1)^{-n / 2}\right\}
$$

and

$$
\left\|T^{\sim}(\varrho)\right\|_{\mathrm{tr}} \leqq e^{c_{n} \lambda t}\|\varrho\|_{\mathrm{tr}}
$$

for all $\varrho \in V$ and $t \geqq 0$. We define $T^{\sim}{ }_{t}$ as the minimal solution of the evolution equation

$$
\varrho^{\prime}(t)=Y \varrho+\varrho Y^{*}+\lambda J^{\sim}(\varrho)
$$

where

$$
Y=-i H-\lambda R / 2
$$

commutes with $N$,

$$
J^{\sim}(\varrho)=\int_{\mathbb{R}^{3}} B^{\sim}{ }_{x} \varrho B_{x}^{\sim *} d x
$$

and

$$
B_{x}^{\sim}=(N+1)^{n / 2} B_{x}(N+1)^{-n / 2} .
$$


The application of $B_{x}$ or $B^{\sim}$ increases the particle number by one, so

$$
\begin{aligned}
& Y+Y^{*}+\lambda \int_{\mathbb{R}^{3}} B_{x}^{* *} B_{x}^{\sim} d x \\
& =-\lambda R+\lambda \int_{\mathbb{R}^{3}}[(N+2) /(N+1)]^{n / 2} B_{x}^{*} B_{x}[(N+2) /(N+1)]^{n / 2} d x \\
& =\lambda R\left\{[(N+2) /(N+1)]^{n}-1\right\} \\
& \leqq \lambda c N\left\{[(N+2) /(N+1)]^{n}-1\right\} \\
& \leqq a 1 .
\end{aligned}
$$

It follows by Theorem 3.6 of [2] that Equation (2.5) is satisfied.

By Theorem 4.1 of [2] and its analogue for Equation (2.6) we see that if $\varrho$ lies in a certain dense subspace $V^{\prime}$ of $V$ and $0 \leqq 2 c t<1$ then

$$
T_{t}(\varrho)=S_{t}(\varrho)+\lambda \int_{s=0}^{t} S_{t-s} J S_{s} \varrho d s+\ldots
$$

and

$$
T^{\sim}(\varrho)=S_{t}(\varrho)+\lambda \int_{s=0}^{t} S_{t-s} J^{\sim} S_{s} \varrho d s+\ldots
$$

where

$$
S_{t}(\varrho)=e^{Y t} \varrho e^{Y * t} .
$$

A term by term comparison establishes that Equation (2.4) is satisfied if $\varrho \in V^{\prime}$ and $0 \leqq 2 c t<1$. Its validity for all $\varrho \in V$ and all $t \geqq 0$ follows by density and use of the semigroup property.

Following the notation of [2] we denote by $Q_{n}$ the projection of $\mathscr{F}$ onto its $n$-particle subspace $\mathscr{F}_{n}$.

\section{Lemma 2.2. If}

$$
\varrho=Q_{k} \varrho Q_{k}
$$

then

$$
\left[T_{t}(\varrho), N\right]=0
$$

for all $\lambda, t \geqq 0$. Moreover

$$
T_{t}(\varrho)=\sum_{m=0}^{\infty} \varrho_{m}(t)
$$

for all $\lambda, t \geqq 0$, where

$$
\varrho_{m}(t)=Q_{m+k} T_{t}(\varrho) Q_{m+k}
$$

satisfies

$$
\left\|\varrho_{m}(t)\right\|_{\mathrm{tr}} \leqq a e^{c_{3} \lambda t}(1+m)^{-3} .
$$

Proof. If $\varrho_{m}(t)$ is defined by Equation (2.17) then

$$
\varrho_{m}(t)=(1+m+k)^{-3} Q_{m+k}(1+N)^{3 / 2} T_{t}(\varrho)(1+N)^{3 / 2} Q_{m+k}
$$


so

$$
\begin{aligned}
\left\|\varrho_{m}(t)\right\|_{\mathrm{tr}} & \leqq(1+m+k)^{-3}\left\|T_{t}(\varrho)\right\|_{3} \\
& \leqq(1+m+k)^{-3} e^{c_{3} \lambda t}\|\varrho\|_{3} \\
& \leqq a(1+m)^{-3} e^{c_{3} \lambda t} .
\end{aligned}
$$

The validity of Equation (2.15) if $[\varrho, N]=0$ and $\varrho \in V^{\prime}$ and $0 \leqq 2 c t<1$ may be deduced directly from Equation (2.10). By the density of $V^{\prime}$ it holds if $[\varrho, N]=0$, $\varrho \in V$ and $0 \leqq 2 c t<1$, and by the semigroup property it holds for all $t>0$. It follows that

$$
\begin{aligned}
T_{t}(\varrho) & =\sum_{m=-k}^{\infty} Q_{k+m} T_{t}(\varrho) \\
& =\sum_{m=-k}^{\infty} \varrho_{m}(t) .
\end{aligned}
$$

We eliminate the negative indices $m$ by obtaining explicit expressions for $\varrho_{m}(t)$ which will be used later. The expressions are essentially just the terms of Equation (2.11) but we need to extend the range of validity of that equation to all $t \geqq 0$. This uses notation and ideas from [2].

If $t \geqq 0$ then

$$
\begin{aligned}
\varrho_{m}(t) & =Q_{k+m}\left\{\lim _{r \rightarrow 1} \lim _{n \rightarrow \infty} T_{t}^{n r}(\varrho)\right\} Q_{k+m} \\
& =\lim _{r \rightarrow 1} \lim _{n \rightarrow \infty}\left\{Q_{k+m} T_{t}^{n r}(\varrho) Q_{k+m}\right\} .
\end{aligned}
$$

Now

$$
T_{t}^{n r}(\varrho)=S_{t}(\varrho)+\lambda r \int_{s=0}^{t} S_{t-s} J_{n} S_{s} \varrho d s+\ldots
$$

for all $t \geqq 0$, the operators $J_{n}$ being bounded approximations to $J$. It follows that if $m<0$

$$
Q_{k+m} T_{t}^{n r}(\varrho) Q_{k+m}=0
$$

while if $m \geqq 0$

$$
Q_{k+m} T_{t}^{n r}(\varrho) Q_{k+m}=\lambda^{m} r^{m} \int_{\Delta^{m}} S_{t-s_{1}} J_{n} S_{s_{1}-s_{2}} \ldots J_{n} S_{s_{m}} \varrho d^{m} S .
$$

We have introduced and will use throughout the paper the contracted notation

$$
s=\left(s_{1}, \ldots, s_{m}\right) \quad d^{m} s=d s_{1} d s_{2} \ldots d s_{m}
$$

and

$$
\int_{\Delta^{m}}=\int_{s_{1}=0}^{t} \int_{s_{2}=0}^{s_{1}} \ldots \int_{s_{m}=0}^{s_{m}-1}
$$

and similarly for the space variables $x_{1}, \ldots, x_{m}$. It follows from Equations (2.22) and (2.25) that

$$
\varrho_{m}(t)=\lambda^{m} \int_{\Delta^{m}} S_{t-s_{1}} J S_{s_{1}-s_{2}} \ldots J S_{s_{m}} \varrho d^{m} S
$$

for all $m \geqq 0$ and $t \geqq 0$, while $\varrho_{m}(t)=0$ if $m<0$.

We turn now to the semigroup $D_{t}$ on $W=\mathscr{T}_{s}(\mathscr{H})$. 
Lemma 2.3. The evolution equation

$$
\sigma^{\prime}(t)=-i[H, \sigma]-\lambda(A \sigma+\sigma A) / 2+\lambda K(\sigma)
$$

on $W$ has the solution

$$
\begin{aligned}
\sigma(t) & =D_{t}\{\sigma(0)\} \\
& =\sum_{m=0}^{\infty} \lambda^{m} \int_{\Delta^{m}} U_{t-s_{1}} K U_{s_{1}-s_{2}} \ldots K U_{s_{m}} \sigma d^{m} s
\end{aligned}
$$

convergent in trace norm for all $t \geqq 0$, where

$$
U_{t}(\sigma)=e^{(-i H-\lambda A / 2) t} \sigma e^{(i H-\lambda A / 2) t} .
$$

Moreover $D_{t}$ is a strongly continuous, positive, one-parameter semigroup on $W$.

Proof. We first show that Equation (1.9) defines a bounded operator $A \geqq 0$ on $\mathscr{H}$. If $f \in L^{2}\left(\mathbb{R}^{3}\right)$ and ${ }^{\wedge}$ denotes the unitary Fourier transform operator then

$$
\begin{aligned}
\langle A f, f\rangle & =\int_{\mathbb{R}^{3}}\left|\left\langle f, f_{x}^{1}\right\rangle\right|^{2} d x \\
& =\int_{\mathbb{R}^{3}}|h(k)|^{2} d k
\end{aligned}
$$

where

$$
\begin{aligned}
h(k) & =(2 \pi)^{-3 / 2} \int_{\mathbb{R}^{3}} f(y) f^{1}(y-x)-e^{-i x \cdot k} d y d x . \\
& =(2 \pi)^{3 / 2} f^{\wedge}(k) f^{1 \wedge}(k)^{-} .
\end{aligned}
$$

Therefore

$$
\langle A f, f\rangle=(2 \pi)^{3} \int_{\mathbb{R}^{3}}\left|f^{\wedge}(k)\right|^{2}\left|f^{1 \wedge}(k)\right|^{2} d k
$$

and $A$ is, in momentum space, the operator of multiplication by the bounded function

$$
a(k)=(2 \pi)^{3}\left|f^{1 \wedge}(k)\right|^{2} .
$$

It follows that Equation (2.31) defines a strongly continuous positive one parameter contraction semigroup $U_{t}$ on $W$. If $\sigma \in W^{+}$then

$$
0 \leqq \operatorname{tr}[K \sigma] \leqq 2 \operatorname{tr}[A \sigma]
$$

by Equation (1.8), so $K$ is a bounded positive linear map on $W$. Therefore Equation (2.30) converges for all $t \geqq 0$ and defines a semigroup $D_{t}$ on $W$ which is positive for all $t \geqq 0$.

The passage between the semigroups $T_{t}$ and $D_{t}$ is effected by introducing yet another semigroup $T_{t}^{-}$which acts on a multi-particle space with MaxwellBoltzmann statistics, namely

$$
\mathscr{F}^{-}=\mathbb{C} \oplus \mathscr{H} \oplus(\mathscr{H} \otimes \mathscr{H}) \oplus \ldots .
$$

The free Hamiltonian $H^{-}$on $\mathscr{F}^{-}$is defined in the obvious way while $R^{-}$is the operator which maps $\otimes^{n} \mathscr{H}$ into itself for all $n$ and is given there by

$$
\begin{aligned}
R^{-}= & A \otimes 1 \otimes 1 \otimes \ldots \otimes 1+1 \otimes A \otimes 1 \otimes \ldots \otimes 1 \\
& +\ldots+1 \otimes 1 \otimes \ldots \otimes 1 \otimes A .
\end{aligned}
$$


We define the one-parameter semigroup $S^{-}$on $V^{-}=\mathscr{T}_{s}\left(\mathscr{F}^{-}\right)$by

$$
S_{t}^{-}(\varrho)=e^{Y^{-*_{t}} \varrho} e^{Y^{\boldsymbol{F}_{*}}}
$$

where

$$
Y^{-}=-i H^{-}-\lambda R^{-} / 2
$$

noting that although $S_{t}^{-}$leaves the subspace $V$ of $V^{-}$invariant, its restriction to $V$ does not coincide with $S_{t}$, defined in Equation (2.13).

If $x \in \mathbb{R}^{3}$ and $m, n$ are integers with $1 \leqq m \leqq n$ we define operators $B_{x m n}^{-}$on $\mathscr{F}^{-}$ as follows. The operator is zero except on $\otimes^{n} \mathscr{H}$ and maps this subspace into $\otimes^{n+1} \mathscr{H}$. The formula

$$
\begin{aligned}
& B_{x m n}^{-}\left(\phi_{1} \otimes \phi_{2} \otimes \ldots \otimes \phi_{n}\right) \\
& =\left\langle\phi_{m}, f_{x}^{1}\right\rangle\left(\phi_{1} \otimes \ldots \otimes \phi_{m-1} \otimes f_{x}^{3} \otimes f_{x}^{2} \otimes \phi_{m+1} \otimes \ldots \otimes \phi_{n}\right)
\end{aligned}
$$

uniquely determines $B_{x m n}^{-}$on $\otimes^{n} \mathscr{H}$. We define the unbounded positive linear $\operatorname{map} J^{-}: V^{-} \rightarrow V^{-}$by

$$
J^{-}(\varrho)=\sum_{m, n} J_{m n}^{-}(\varrho)
$$

where

$$
J_{m n}^{-}(\varrho)=\int_{\mathbb{R}^{3}} B_{x m n}^{-} B_{x m n}^{-*} d x .
$$

It may be seen that

$$
\operatorname{tr}\left[J^{-}(\varrho)\right]=\operatorname{tr}[R \varrho]
$$

for those $\varrho \in V^{-}$for which both sides make sense. We define

$$
V_{n}^{-}=\left\{\varrho \in V^{-}:\|\varrho\|_{n} \equiv \operatorname{tr}\left[(1+N)^{n} \varrho \varrho \mid\right]<\infty\right\}
$$

and we define a projection $P^{-}: V^{-} \rightarrow W$ by

$$
P^{-}=\sum_{m, n} P_{m n}^{-}
$$

where $P_{m n}^{-}$is as follows. If $Q_{n}^{-}$is the projection of $\mathscr{F}^{-}$onto its $n$-particle subspace $\mathscr{F}_{n}$ then

$$
P_{m n}^{-}(\varrho)=P_{m n}^{-}\left(Q_{n} \varrho Q_{n}\right) .
$$

For states with support in $\mathscr{F}_{n}^{-}, P_{m n}^{-}$is determined by

$$
\begin{aligned}
& P_{m n}^{-}\left(\left|\phi_{1} \otimes \ldots \otimes \phi_{n}\right\rangle\left\langle\phi_{1} \otimes \ldots \otimes \phi_{n}\right|\right) \\
& =\left|\phi_{m}\right\rangle\left\langle\phi_{m}\right| \cdot \prod_{r \neq m}\left\|\phi_{r}\right\|^{2}
\end{aligned}
$$

if $1 \leqq m \leqq n$, and $P_{m n}^{-}=0$ otherwise.

Lemma 2.4. The minimal solution of the equation

$$
\varrho^{\prime}(t)=-i\left[H^{-}, \varrho\right]-\lambda\left(R^{-} \varrho+\varrho R^{-}\right) / 2+J^{-}(\varrho)
$$


where $\varrho(t) \in V^{-}$, is trace preserving and determines a semigroup $T_{t}^{-}$on $V^{-}$. This semigroup leaves each of the subspaces $V^{-}$invariant and there are constants such that

$$
\left\|T^{-} \varrho\right\|_{n} \leqq e^{c_{n} \lambda t}\|\varrho\|_{n}
$$

for all $\varrho \in V^{-}$and all $\lambda, t \geqq 0$. If $\varrho=Q_{k} \varrho Q_{k}$ then

$$
T^{-}(\varrho)=\sum_{m=0}^{\infty} \varrho_{m}^{-}(t)
$$

for all $\lambda, t \geqq 0$ where

$$
\varrho_{m}^{-}(t)=\lambda^{m} \int_{\Delta^{m}} S_{t-s_{1}}^{-} J^{-} S_{s_{1}-s_{2}}^{-} \ldots J^{-} S_{s_{m}}^{-} \varrho d^{m} S
$$

satisfies

$$
\left\|\varrho_{m}^{-}(t)\right\|_{\mathrm{tr}} \leqq a e^{c_{3} \lambda t}(1+m)^{-3}
$$

for all $\lambda, t \geqq 0$ and all integers $m$.

Proof. We omit this since it is so similar to the proofs of Lemmas 2.1 and 2.2.

The following lemma explains the introduction of the semigroup $T^{-}$.

Lemma 2.5. If $f \in \mathscr{H}$ and $\varrho \in W$ is defined as

$$
\varrho=|f\rangle\langle f|
$$

then

$$
P^{-} T^{-}{ }_{t} \varrho=D_{t} \varrho
$$

for all $t \geqq 0$.

Proof. Applying Equations (2.42) and (2.46) to Equation (2.52) leads to

$$
P^{-} Q_{m}^{-}(t)=\sum_{|g|=m} \int_{\Delta^{m}} \mu^{g}(s) d^{m} S
$$

where $\mu^{g}(s) \in W^{+}$and the sum is over all connected graphs $g$ obtainable from

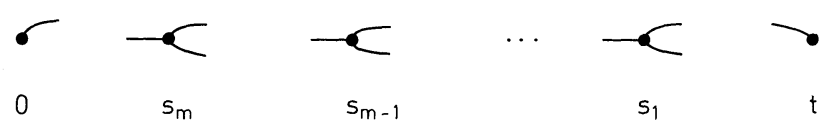

where each vertex has been labelled with the appropriate time and the number of vertices is $(|g|+2)$. In a connected graph $g$ the left hand vertex denotes the initial state $\varrho$, the other vertices from left to right determine which of the $J_{m n}^{-}$are chosen and the right hand vertex determines which of the $P_{m n}$ is chosen. Thus for the graph

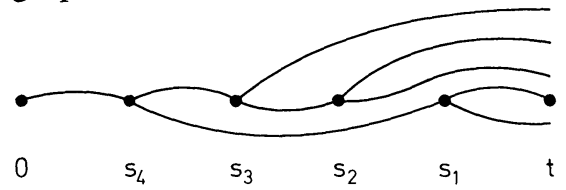


we have

$$
\mu^{g}(s)=P_{45}^{-} S_{t-s_{1}}^{-} J_{44}^{-} S_{s_{1}-s_{2}}^{-} J_{23}^{-} S_{s_{2}-s_{3}}^{-} J_{12}^{-} S_{s_{3}-s_{4}}^{-} J^{-}{ }_{11} S_{s_{4}}^{-} Q .
$$

By Equations (2.51) and (2.56) we find that for all $\lambda, t \geqq 0$

$$
P^{-} T_{t}^{-} \varrho=\sum_{k} v^{k}(t)
$$

where

$$
v^{k}(t)=\sum_{\{g: \dot{g}=k\}} \int_{\Delta^{|g|}} \mu^{g}(s) d^{|g|} S
$$

and the skeleton $\dot{g}$ of a graph $g$ is defined as the smallest connected subgraph containing both end vertices of $g$.

Every graph $g$ may be written as the disjoint union of its skeleton $k$ and connected subgraphs $g_{1}, \ldots, g_{n}$, one associated with every internal vertex of $k$. One may use this decomposition to obtain a new expression

$$
v^{k}(t)=\int_{\Delta^{n}} \sigma^{k}(s)\left\{\prod_{r=1}^{n} h_{r}\left(s_{r}\right)\right\} d^{n} s
$$

where $n$ is the number of internal vertices of $k$. In this equation the scalar $h_{r}\left(s_{r}\right)$ is a sum over all possible connected subgraphs $g_{r}$ associated with the $r$-th internal vertex of $k$, as $g$ varies. By inspection

$$
h_{r}\left(s_{r}\right)=\operatorname{tr}\left[T_{t-s_{r}}^{-}\{|\psi\rangle\langle\psi|\}\right]
$$

where $\psi$ is $f_{x}^{2}$ or $f_{x}^{3}$ or $f$, and in any case has unit norm. Since $T_{t}^{-}$is trace preserving

$$
h_{r}\left(s_{r}\right)=1 \text {. }
$$

The term $\sigma^{k}(s) \in W^{+}$is given by

$$
\sigma^{k}(s)=\lambda^{n} U_{t-s_{1}} K_{\alpha_{1}} U_{s_{1}-s_{2}} \ldots K_{\alpha_{n}} U_{s_{n}} \varrho
$$

where $n$ and $\alpha_{1}, \ldots, \alpha_{n}$ depend on the graph $k$.

The proof is completed by comparing the last six equations with Equation (2.30).

Theorem 2.6. If $f \in \mathscr{S}$ let $\varrho \in W^{+}$be defined by

$$
\varrho=|f\rangle\langle f| \text {. }
$$

Then

$$
\lim _{\lambda \rightarrow 0}\left\{\sup _{0 \leqq \lambda t \leqq \tau_{0}}\left\|P T_{t} \varrho-D_{t} \varrho\right\|_{\operatorname{tr}}\right\}=0
$$

for all $0 \leqq \tau_{0} \neq \infty$.

Proof. By Lemmas 2.2, 2.4, and 2.5, the bracketed expression in Equation (2.67) is less than or equal to

$$
\sum_{m=0}^{\infty}\left\{\sup _{0 \leqq \lambda t \leqq \tau_{0}}\left\|P \varrho_{m}(t)-P^{-} \varrho_{m}^{-}(t)\right\|_{\mathrm{tr}}\right\} .
$$


Since $\varrho_{m}(t)$ and $\varrho_{m}^{-}(t)$ both have support in the $m$-particle space, Equations (2.18) and (2.53) imply that if $0 \leqq \lambda t \leqq \tau_{0}$ then

$$
\left\|P \varrho_{m}(t)-P^{-} \varrho_{m}^{-}(t)\right\|_{\mathrm{tr}} \leqq c(1+m)^{-2}
$$

where $c$ depends on $\tau_{0}$. To prove the theorem it is therefore sufficient to show that for fixed $m$

$$
\lim _{\lambda \rightarrow 0}\left\{\sup _{0 \leqq \lambda t \leqq \tau_{0}}\left\|P \varrho_{m}(t)-P^{-} \varrho_{m}^{-}(t)\right\|_{\mathrm{tr}}\right\}=0 \text {. }
$$

We do this in two stages. If $\lambda, t \geqq 0$ we define

$$
\varrho_{m}^{\sim}(t)=\lambda^{m} \int_{\Delta^{m}} S_{t-s_{1}}^{-} J S_{s_{1}-s_{2}}^{-} \ldots J S_{s_{m}}^{-} \varrho d^{m} s .
$$

In Theorem 3.2 we prove that

$$
\lim _{\lambda \rightarrow 0}\left\{\sup _{0 \leqq \lambda t \leqq \tau_{0}}\left\|P \varrho_{m}(t)-P \varrho_{m}^{\sim}(t)\right\|_{\operatorname{tr}}\right\}=0
$$

while in Theorem 3.3 we prove that

$$
\lim _{\lambda \rightarrow 0}\left\{\sup _{0 \leqq \lambda t \leqq \tau_{0}}\left\|P \varrho_{m}^{\tilde{m}}(t)-P^{-} \varrho_{m}^{-}(t)\right\|_{\mathrm{tr}}\right\}=0 \text {. }
$$

These together yield Equation (2.70) and so complete the proof.

It is explained in [2] how one may include in Equation (1.1) further terms representing neutron-atom scattering, neutron absorption and external neutron sources, the last having to be restricted to a finite volume in order to stay within the Fock sector. One may also allow the initial state $\varrho$ to be more general than we have indicated. The statement and proof of Theorem 2.6 require only the expected changes. The theorem is however limited to fermion fields because of our reliance on Proposition 5.2 of [2].

\section{§ 3. The Diagrammatic Analysis}

The operator $R^{-}$on $\mathscr{F}^{-}$defined in Equation (2.38) leaves $\mathscr{F}$ invariant and on that subspace is given by

$$
R^{-}=\int_{\mathbb{R}^{3}} a^{*}\left(f_{x}^{1}\right) a\left(f_{x}^{1}\right) d x .
$$

It follows that the operator $Y^{-}$restricted to $\mathscr{F}$ is quadratic in the creation and annihilation operators. Therefore

$$
e^{Y \bar{t}} a^{*}(\phi) e^{-Y^{\top} t}=a^{*}\left(e^{(-i H-\lambda A / 2) t} \phi\right)
$$

for all $\phi \in \mathscr{H}$ and $t \in \mathbb{R}$. In order to use Wick's theorem we need some estimates on the two-point functions

$$
F_{i}(x, t)=\left\langle e^{(-i H-\lambda A / 2) t} g_{x}, g^{i}\right\rangle
$$

where $g, g^{1}, g^{2} \in \mathscr{S}, x \in \mathbb{R}^{3}$ and $t \geqq 0$.

Lemma 3.1. If $0 \leqq \lambda t \leqq \tau_{0}$ then

$$
\int_{\mathbb{R}^{3}}\left|F_{1}(x, t)\right|^{2} d x \leqq c
$$


and

$$
\left|F_{1}(x, t)\right| \leqq c(1+t)^{-3 / 2} .
$$

If $g$ and $g^{1}$ have disjoint supports in momentum space then

$$
F_{1}(x, t)=0 \text {. }
$$

If $0 \leqq \lambda t \leqq \tau_{0}$ and $0 \leqq \lambda s \leqq \tau_{0}$ then

$$
\left|\int_{\mathbb{R}^{3}} F_{1}(x, t) F_{2}(x, s)-d x\right| \leqq\left. c(1+|s-t|)^{-3 / 2}\right|^{2} d k
$$

while if $g^{1}$ and $g^{2}$ have disjoint supports in momentum space

$$
\int_{\mathbb{R}^{3}} F_{1}(x, t) F_{2}(x, s)-d x=0 .
$$

Proof. By Equation (2.35) and Fourier analysis we obtain

$$
F_{1}(x, t)=\int_{\mathbb{R}^{3}} e^{\left(-i k^{2}-\lambda a(k) / 2\right) t} g(k) g^{1}(k)-e^{i k \cdot x} d k
$$

so by the Plancherel theorem

$$
\begin{aligned}
\int_{\mathbb{R}^{3}}\left|F_{1}(x, t)\right|^{2} d x & =\int_{\mathbb{R}^{3}}\left|e^{\left(-i k^{2}-\lambda a(k) / 2\right) t} g(k) g^{1}(k)-\right|^{2} d k \\
& \leqq e^{\|a\|_{\infty} \tau_{0}}\|g\|_{2}^{2}\left\|g^{1}\right\|_{\infty}^{2} .
\end{aligned}
$$

This proves Equation (3.4), and Equation (3.6) is an immediate consequence of Equation (3.9).

We next define $g^{\tau}$ by

$$
g^{\tau}=e^{-A \tau / 2} g^{1}
$$

so that by Equation (2.35) and Fourier analysis

$$
\left\{g^{\tau}: 0 \leqq \tau \leqq \tau_{0}\right\}
$$

is a compact set in $\mathscr{S}$ for the usual topology of $\mathscr{S}$. Therefore

$$
\sup \left\{\left\|g^{\tau}\right\|_{1}: 0 \leqq \tau \leqq \tau_{0}\right\}<\infty .
$$

Therefore if $0 \leqq \lambda t \leqq \tau_{0}$

$$
\begin{aligned}
\left|F_{1}(x, t)\right| & =\left|(4 \pi i t)^{-3 / 2} \int_{\mathbb{R}^{6}} e^{-(y-z)^{2} / 4 i t} g_{x}(y) g^{\lambda t}(z)-d y d z\right| \\
& \leqq c t^{-3 / 2}\left\|g_{x}\right\|_{1}\left\|g^{\lambda t}\right\|_{1} \\
& \leqq c t^{-3 / 2} .
\end{aligned}
$$

Combining this with the estimate

$$
\left|F_{1}(x, t)\right| \leqq c\|g\|_{2}\left\|g^{1}\right\|_{2}
$$

derived from Equation (3.9) yields Equation (3.5). 
From Equation (3.9) and the Plancherel theorem we obtain

$$
\begin{aligned}
& \int_{\mathbb{R}^{3}} F_{1}(x, t) F_{2}(x, s)-d x \\
& =(2 \pi)^{3} \int_{\mathbb{R}^{3}} e^{\left(-i k^{2}-\lambda a(k) / 2\right) t} g(k) g^{1}(k)-e^{\left(i k^{2}-\lambda a(k) / 2\right) s} g(k)-g^{2}(k) d k .
\end{aligned}
$$

This is estimated in a similar fashion to obtain Equations (3.7) and (3.8).

We shall also use the following method of estimating trace norms of operators defined initially as quadratic forms.

Lemma 3.2. If $h$ is a function defined on $\mathbb{R}^{3 m}$ and

$$
\langle\sigma \phi, \psi\rangle=\int_{\mathbb{R}^{3 m}} h(x)\left\langle\phi, \phi\left(x_{m}\right)\right\rangle\left\langle\psi\left(x_{m}\right), \psi\right\rangle d^{m} x
$$

for all $\phi, \psi \in \mathscr{H}$ then

$$
\|\sigma\|_{\mathrm{tr}} \leqq \int_{\mathbb{R}^{3}} \int_{\mathbb{R}^{3(m-1)}} h(x) d^{m-1} x \mid\left\|\phi\left(x_{m}\right)\right\|\left\|\psi\left(x_{m}\right)\right\| d x_{m} .
$$

If

$$
\langle\sigma \phi, \psi\rangle=\int_{\mathbb{R}^{3 m}} h(x)\left\langle\phi, \phi\left(x_{m-1}\right)\right\rangle\left\langle\psi\left(x_{m}\right), \psi\right\rangle d^{m} x
$$

for all $\phi, \psi \in \mathscr{H}$ then

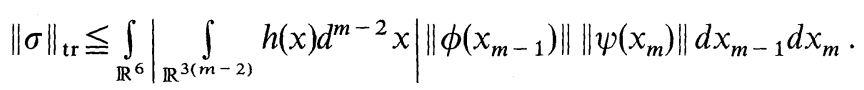

Proof. For the first part if

$$
g\left(x_{m}\right)=\int_{\mathbb{R}^{3(m-1)}} h(x) d^{m-1} x
$$

then

$$
\sigma=\int_{\mathbb{R}^{3}} g\left(x_{m}\right)\left|\phi\left(x_{m}\right)\right\rangle\left\langle\psi\left(x_{m}\right)\right| d x_{m}
$$

so

$$
\|\sigma\|_{\mathrm{tr}} \leqq \int_{\mathbb{R}^{3}}\left|g\left(x_{m}\right)\right|\left\|\phi\left(x_{m}\right)\right\|\left\|\psi\left(x_{m}\right)\right\| d x_{m} .
$$

The other part is proved similarly.

Theorem 3.2. If $0 \leqq \tau_{0}<\infty$ then

$$
\lim _{\lambda \rightarrow 0}\left\{\sup _{0 \leqq \lambda t \leqq \tau_{0}}\left\|P \varrho_{m}(t)-P \varrho_{m}^{\tilde{m}}(t)\right\|_{\mathrm{tr}}\right\}=0 \text {. }
$$

Proof. From Equations (1.3), (1.4), (3.1) and the anti-commutation relations we obtain

$$
R=R^{-}+R_{1}+R_{2}+R_{3}
$$


where

$$
\begin{aligned}
& R_{1}=-\int_{\mathbb{R}^{3}} a^{*}\left(f_{x}^{1}\right) a^{*}\left(f_{x}^{2}\right) a\left(f_{x}^{2}\right) a\left(f_{x}^{1}\right) d x \\
& R_{2}=-\int_{\mathbb{R}^{3}} a^{*}\left(f_{x}^{1}\right) a^{*}\left(f_{x}^{3}\right) a\left(f_{x}^{3}\right) a\left(f_{x}^{1}\right) d x
\end{aligned}
$$

and

$$
R_{3}=\int_{\mathbb{R}^{3}} a^{*}\left(f_{x}^{1}\right) a^{*}\left(f_{x}^{2}\right) a^{*}\left(f_{x}^{3}\right) a\left(f_{x}^{3}\right) a\left(f_{x}^{2}\right) a\left(f_{x}^{1}\right) d x .
$$

It follows by Equations (2.7) and (2.40) that if $\lambda, t \geqq 0$ then

$$
S_{t}(\varrho)=S_{t}^{-}(\varrho)+\lambda \int_{s=0}^{t} S_{t-s} \mathscr{C} S_{s}^{-} \varrho d s
$$

where the operator $C$ on $V$ is defined by

$$
C(\varrho)=-\frac{1}{2} \sum_{i=1}^{3}\left(R_{i} \varrho+\varrho R_{i}\right) .
$$

By Equations (2.28), (2.71), and (3.29) we now obtain

$$
\begin{aligned}
& \varrho_{m}(t)-\varrho_{m}^{\sim}(t) \\
& =\lambda^{m+1} \int_{\Delta^{m+1}} S_{t-s_{1}} J S_{s_{1}-s_{2}} J \ldots J S_{s_{m}-s_{m+1}} C S_{s_{m+1}}^{-} \varrho d^{m+1} s \\
& \quad+\lambda^{m+1} \int_{\Delta^{m+1}} S_{t-s_{1}} J S_{s_{1}-s_{2}} J \ldots S_{s_{m-1}-s_{m}} C S_{s_{m}-s_{m+1}}^{-} J S_{s_{m+1}}^{-} \varrho d^{m+1} s+\ldots \\
& \quad+\lambda^{m+1} \int_{\Delta^{m+1}} S_{t-s_{1}} C S_{s_{1}-s_{2}}^{-} J S_{s_{2}-s_{3}}^{-} J \ldots J S_{s_{m+1}}^{-} \varrho d^{m+1} s .
\end{aligned}
$$

Crude norm estimates of the part of each integrand to the left of the operator $C$ lead to

$$
\begin{aligned}
& \left\|P \varrho_{m}(t)-P \varrho_{m}^{\sim}(t)\right\|_{\mathrm{tr}} \\
& \leqq c \lambda^{m+1} \sum_{r=1}^{m+1} \int_{\Delta^{m+1}}\left\|C S_{s_{r}-s_{r+1}}^{-} J S_{s_{r+1}-s_{r+2}}^{-} J \ldots J S_{s_{m+1}}^{-} \varrho\right\|_{\mathrm{tr}} d^{m+1} S .
\end{aligned}
$$

To prove the theorem it is sufficient to show that if $0 \leqq \lambda t \leqq \tau_{0}$ and $1 \leqq r \leqq m+1$ then

$$
I_{\lambda}=\lambda^{r} \int_{\Delta^{r}}\left\|C S_{s_{r}}^{-} J S_{s_{r-1}-s_{r}}^{-} J S_{s_{r-2}-s_{r-1}}^{-} J \ldots J S_{s_{1}-s_{2}}^{-} \sigma\right\|_{\mathrm{tr}} d{ }^{r} S
$$

converges to zero as $\lambda \rightarrow 0$.

The first step is to convert the expression for $I_{\lambda}$ into a Hilbert space integral by using Equations (1.2), (2.39), (2.66), and (3.30). We obtain

$$
\begin{aligned}
& I_{\lambda} \leqq \sum_{i=1}^{3} \lambda^{r} \int_{\Delta^{r}} \int_{\mathbb{R}^{3(r-1)}} \| R_{i} e^{Y-s_{r}} B_{x_{r}} e^{Y-\left(s_{r}-1-s_{r}\right)} \ldots \\
& B_{x_{2}} e^{Y^{-}\left(s_{1}-s_{2}\right)} f\|\cdot\| e^{Y-s_{r}} B_{x_{r}} e^{Y^{-}\left(s_{r-1}-s_{r}\right)} \ldots B_{x_{2}} e^{Y^{-\left(s_{1}-s_{2}\right)} f \| d^{c} s d^{c-1} x}
\end{aligned}
$$

where $x=\left(x_{2}, \ldots, x_{r}\right)$. If we define

$$
J_{\lambda, i}=\int_{\Delta^{r}} \int_{\mathbb{R}^{3(r-1)}}\left\|R_{i} e^{Y^{-s_{r}}} \ldots f\right\|^{2} d^{r} s d^{r-1} x
$$


and

then

$$
J_{\lambda}=\int_{\Delta^{r}} \int_{\mathbb{R}^{3(r-1)}}\left\|e^{Y s_{r}} \ldots f\right\|^{2} d^{r} s d^{r-1} x
$$

$$
I_{\lambda} \leqq \sum_{i=1}^{3} \lambda^{r}\left\{J_{\lambda, i} J_{\lambda}\right\}^{1 / 2} .
$$

If

$$
E=a^{*}(f)
$$

and

$$
F_{x}=a^{*}\left(f_{x}^{1}\right) a^{*}\left(f_{x}^{2}\right) a\left(f_{x}^{2}\right) a\left(f_{x}^{1}\right)
$$

and

$$
X_{s}=e^{Y \bar{s}} X e^{-Y \bar{s}}
$$

for any operator $X$ on $\mathscr{F}$, then

$$
\begin{aligned}
& J_{\lambda, 1}=\int_{\Delta^{r}} \int_{\mathbb{R}^{3(r+1)}}\left\langle E_{s_{1}}^{*} B_{x_{2} s_{2}}^{*} B_{x_{3} s_{3}}^{*} \ldots\right. \\
& \left.B_{x_{r} s_{r}}^{*} F_{y}^{*} F_{z} B_{x_{r} s_{r}} \ldots B_{x_{2} s_{2}} E_{s_{1}}\right\rangle_{\text {vac }} d^{r} s d^{r-1} x d y d z .
\end{aligned}
$$

By Equation (3.2) and Wick's theorem this may be expressed as a sum over all possible contractions of graphs of the type
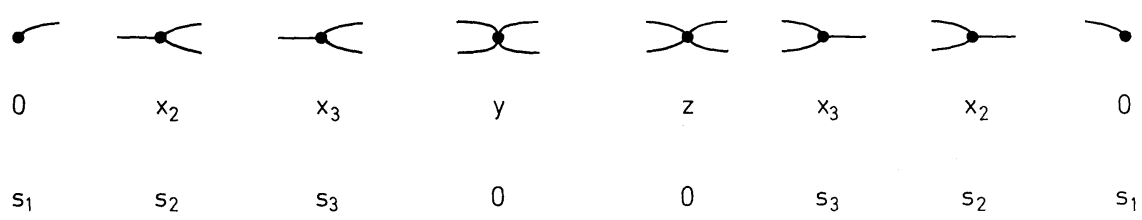

whose vertices have been labelled by the appropriate space and time coordinates. The integrand associated to each fully contracted graph is the product of the contributions of each of its legs, which are estimated using Lemma 3.1.

We use the legs of the triple vertices which point away from the centre to control the space integrations with respect to $x_{2}, \ldots, x_{r}$ uniformly with respect to time. We use the left-hand legs of the two quadruple vertices to control the $y, z$ integrations uniformly with respect to time. We finally use one of the righthand legs of the right-hand quadruple vertex to obtain time decay uniformly in the space variables. The result is that

$$
J_{\lambda, 1} \leqq c t^{r-1} .
$$

We can obtain similar estimates for $J_{\lambda, 2}$ and $J_{\lambda, 3}$, and also

$$
J_{\lambda} \leqq c t^{r} .
$$

Hence

$$
\begin{aligned}
I_{\lambda} & \leqq c \lambda^{r} t^{r-1 / 2} \\
& \leqq c \tau_{0}^{r-1 / 2} \lambda^{1 / 2}
\end{aligned}
$$

which converges to zero as $\lambda \rightarrow 0$. 
Theorem 3.3. If $0 \leqq \tau_{0}<\infty$ then

$$
\lim _{\lambda \rightarrow 0}\left\{\sup _{0 \leqq \lambda t \leqq \tau_{0}}\left\|P \varrho_{m}^{\sim}(t)-P^{-} \varrho_{m}^{-}(t)\right\|_{\mathrm{tr}}\right\}=0 \text {. }
$$

Proof. If $\psi \in \mathscr{H}$ then by Equation (1.12)

$$
\left\langle P \varrho_{m}^{\sim}(t) \psi, \psi\right\rangle=\lambda^{m} \int_{\mathbb{R}^{3 m}} \int_{\Delta^{m}} I(x, s) d^{m} x d^{m} S
$$

where

$$
I(x, s)=\left\langle E_{t}^{*} B_{x_{1} s_{1}}^{*} \ldots B_{x_{m} s_{m}}^{*} a^{*}(\psi) a(\psi) B_{x_{m} s_{m}} \ldots B_{x_{1} s_{1}} E_{t}\right\rangle_{\mathrm{vac}} .
$$

By Wick's theorem

$$
I(x, s)=\sum_{g \in G} I^{g}(x, s)
$$

where $G$ is the set of all complete contractions $g$ of the graph

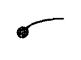

0

t
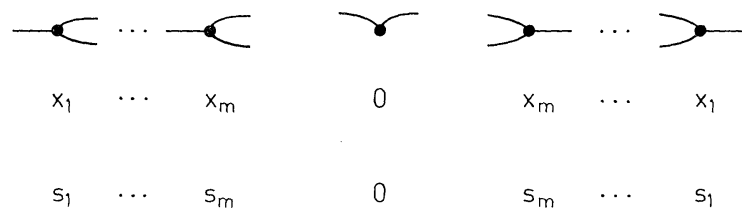

in which each vertex has been labelled with the appropriate space-time coordinate. Moreover $I^{g}(x, s)$ is the product of the two-point functions associated to each link of the graph $g$, with an appropriate sign.

Corresponding to Equation (3.49) there is a decomposition

$$
P \varrho_{m}^{\tilde{m}}(t)=\sum_{g \in G} \sigma_{m}^{g}(t)
$$

where

$$
\left\langle\sigma_{m}^{g}(t) \psi, \psi\right\rangle=\lambda^{m} \int_{\mathbb{R}^{3 m}} \int_{\Delta^{m}} I^{g}(x, s) d^{m} x d^{m} S
$$

for all $\psi \in \mathscr{H}$. Now the one-one correspondence between the set $F$ of all $g$ which are symmetrical with respect to reflection about the centre, and the set of all graphs in the sense of Lemma 2.5, leads to the formula

$$
P^{-} \varrho_{m}^{-}(t)=\sum_{g \in F} \sigma_{m}^{g}(t) \text {. }
$$

We are therefore left with showing that if $g \notin F$ then $\sigma_{m}^{g}$ is asymptotically negligible in trace norm as $\lambda \rightarrow 0$.

The space-time decay of $I^{g}(x, s)$ is estimated by the use of Lemma 3.1. The outgoing legs of each triple vertex are used to control the space integrations uniformly with respect to time by Equation (3.4). The two legs of the central vertex cannot be used at all because they are needed to control the trace norm by Lemma 3.2. The remaining legs must cross from one side of the graph to the other.

These considerations already lead to the estimate

$$
\left\|\sigma_{m}^{g}(t)\right\|_{\mathrm{tr}} \leqq c \lambda^{m} t^{m} \leqq c \tau_{0}^{m}
$$


so to prove $\sigma_{m}^{g}(t)$ is asymptotically negligible we have only to obtain some further control of the time decay of the integrand $I^{g}(x, s)$ when $g$ is not symmetrical.

Graphical considerations show that if $g$ is not symmetrical one of the following things must occur:

(i) One of the legs passing from one side of the graph to the other joins vertices which are not opposite each other.

(ii) One of the legs passing from one side of the graph to the other joins unlike legs of two opposite vertices.

(iii) There is a pair of opposite vertices neither of which is linked directly to the centre, such that the legs from these vertices which go away from the centre are joined to vertices which are not opposite to each other.

(iv) There is a pair of opposite vertices neither of which is linked directly to the centre, such that the legs from these vertices which go away from the centre are joined to unlike legs of two opposite vertices.

In case (i) the extra time decay comes from Equation (3.5). In case (ii) the term vanishes identically by Equation (3.6) while in case (iv) it vanishes identically by Equation (3.8). In case (iii) the extra time decay comes from Equation (3.7); we remark that the significance of the pair of opposite vertices not being linked directly to the centre is that by Lemma 3.2 we can then carry out the corresponding space integration before taking absolute values.

\section{References}

1. Davies, E. B.: Markovian master equations. Commun. math. Phys. 39, 91-110 (1974)

2. Davies, E. B.: Quantum dynamical semigroups and the neutron diffusion equation. Rept. Math. Phys., to appear

3. Davies, E.B., Eckmann,J.-P.: Time decay for fermion systems with persistent vacuum. Helv. Phys. Acta 48, 731-742 (1975)

4. Henin, F.: Entropy, dynamics and molecular chaos, Kac's model. Physica 77, 220 -246 (1974)

5. Kac, M.: Probability and related topics in the physical sciences. Wiley: Interscience 1959

6. McKean,H.P. Jr.: An exponential formula for solving Boltmann's equation for a Maxwellian gas. J. Comb. Theory 2, 358-382 (1967)

7. Martin,P., Emch, G.G.: A rigorous model sustaining van Hove's phenomenon. Helv. Phys. Acta 48, 59-78 (1975)

8. Pulè, J.V.: The Bloch equations. Commun. math. Phys. 38, 241-256 (1974)

Communicated by J. Glimm

Received August 10, 1976 